\title{
Guanine-Deaminase Activity in Rat Brain and Liver
}

\author{
By S. KUMAR, K. K. TEWARI AND P.S. KRISHNAN \\ Department of Biochemistry, University of Lucknow, Lucknow, U.P., India
}

(Received 20 July 1964)

\begin{abstract}
1. Guanine deaminase in rat brain and liver was distributed among all the subcellular fractions: nuclei, 'heavy' mitochondria, 'light' mitochondria, microsomes and the supernatant fluid. The greater part of the activity passed into the soluble fraction. Among the particulate components, the 'light' mitochondria constituted the richest fraction. 2. The sum of the enzymic activities of the component fractions obtained on differential centrifugation was considerably greater than the activity of guanine deaminase in the whole homogenate. 3. The 'heavy'-mitochondrial fraction had a powerful inhibitory effect on the guanine-deaminase activity of the supernatant fraction. 4. All the sedimented fractions, except the microsomes, gave rise to higher guanine-deaminase activity on treatment with Triton X-100. 5. The inhibitory capacity of the 'heavy' mitochondria increased on treatment with Triton X-100; the detergent-treated nuclear fraction also brought about inhibition of the $5000 \mathrm{~g}$ supernatant. 6. Guanine-deaminase inhibitor from the 'heavy' mitochondria was solubilized by high-speed grinding of the particles, followed by treatment with Triton X-100. The inhibitor appeared to be protein in nature, since it was precipitated by trichloroacetic acid and by half-saturation with ammonium sulphate, and was non-diffusible. It was inactivated by heating at $50^{\circ}$ for $5 \mathrm{~min}$. 7 . It is possible that the guanine deaminase associated with particles differs from the soluble enzyme in its response to inhibitor.
\end{abstract}

de Lamirande, Allard \& Cantero (1958) concluded that guanine deaminase of rat liver is present exclusively in the soluble fraction, since the activity of homogenates in iso-osmotic sucrose could be recovered practically quantitatively in the $140000 \mathrm{~g}$ supernatant. No activity could be demonstrated in the whole sedimentable fraction. Talwar, Geol, Mansoor \& Panda (1961) reported the presence of considerable concentration of guaninedeaminase activity in rat brain, as analysed in the $17000 \mathrm{~g}$ supernatants of homogenates prepared in water. The whole homogenate of brain and the residue obtained during centrifugation were not examined for enzymic activity, nor was a localization study carried out with sucrose medium. The present authors find that the distribution of guanine deaminase both in brain and liver presents a complex picture.

\section{MATERIALS AND METHODS}

Cell fractionation. Adult male rats were used in the experiments and were killed by cervical dislocation followed by decapitation. Brain (consisting of olfactory lobes, cerebral hemispheres, corpus callosum, optic lobes and cerebellum) and liver were excised as quickly as possible, cleaned free of extraneous material and used fresh. The
$10 \%(\mathrm{w} / \mathrm{v})$ homogenates of the whole brain and the middle lobe of liver were prepared in $0.25 \mathrm{M}$-sucrose solution by grinding for two lmin. periods with an interval of $1 \mathrm{~min}$. in a Potter-Elvehjem-type homogenizer (size B) made up of glass tube and Teflon pestle (supplied by Arthur H. Thomas Co., Philadelphia, Pa., U.S.A.). The clearance between the pestle and tube, specified by the makers, was $0 \cdot 005-$ $0.007 \mathrm{in}$. The downward and upward movement of the pestle was made $20-25$ times, the contents being held chilled in a bath of ice. For cell fractionation, the homogenate $(\mathrm{H})$ was centrifuged at $700 \mathrm{~g}$ for $10 \mathrm{~min}$. at $0-1^{\circ}$ and the supernatant decanted. The 'nuclear' sediment (N) was suspended in $0.25 \mathrm{M}$-sucrose by operating the homogenizer for $20 \mathrm{sec}$. and the particles were collected by centrifugation as before. This washing process was repeated twice. The combined supernatant and washings was centrifuged at $5000 \mathrm{~g}$ for $20 \mathrm{~min}$. The 'heavy-mitochondrial' sediment $\left(M_{1}\right)$ was washed with $0.25 \mathrm{M}$-sucrose, each time by gentle suspension with the aid of a glass rod, till the final washing (three washings were necessary) was free of guanine-deaminase activity. The wash fluids added to the main supernatant constituted 'soluble fraction $\mathrm{S}_{1}$ '. 'Light mitochondria', inclusive of 'fluffy layer' $\left(\mathrm{M}_{2}\right)$, were sedimented by centrifugation of $S_{1}$ fraction at $15000 \mathrm{~g}$ for lhr. and washed once with the sucrose solution. The supernatant mixed with the wash fluid constituted 'soluble fraction $\mathrm{S}_{2}$ '. All the above operations were carried out in an International model PR-2 refrigerated centrifuge. A further sedimentation was effected by centrifugation of $\mathrm{S}_{2}$ 
fraction at $140000 \mathrm{~g}$ for $1 \mathrm{hr}$. in a Spinco model $\mathrm{L}$ ultracentrifuge, rotor no. 40. The microsomal pellet $\left(\mathbf{M}_{3}\right)$ was not washed. The final supernatant was designated 'soluble fraction $\mathrm{S}_{3}$ '. In some experiments the homogenate was centrifuged directly at $5000 \mathrm{~g}$ to give a sediment $\left(R_{1}\right)$ and the $S_{1}$ fraction, from which the light mitochondria and the microsomal fraction $\left(\mathbf{M}_{3}\right)$ were subsequently separated. In some, the homogenate was initially centrifuged at $15000 \mathrm{~g}$ to yield a sediment $\left(R_{2}\right)$ and the soluble fraction corresponding to fraction $S_{2}$ which was then centrifuged at $140000 \mathrm{~g}$ to yield fraction $S_{3}$. In other experiments, the whole homogenate was directly centrifuged at $140000 \mathrm{~g}$ to give a total sediment $\left(R_{3}\right)$ and supernatant fraction $S_{3}$. The sedimented fractions were dispersed in $0.25 \mathrm{M}$-sucrose before assay.

For treatment with Triton X-100, the detergent was added (final concn. $1 \%, v / v$ ) to samples of the whole homogenate or the separated fractions and allowed to stand for $2 \mathrm{hr}$. at $4^{\circ}$. The conditions chosen for detergent action were selected arbitrarily and may not have been the optimum for maximum exposure of enzyme or inhibitor.

Characterization of cell fractions. The use of the terms 'nuclear', 'heavy-mitochondrial', 'light-mitochondrial' and 'microsomal' fractions in the preceding section was based on the generally accepted pattern of sedimentation on differential centrifugation of homgenates. In view of the fact that brain tissue is less well characterized than liver with respect to the differential centrifugation of the organelles, the brain fractions obtained were subjected, in separate experiments, to biochemical characterization, involving the determination of DNA, RNA and succinoxidase, in addition to total protein. DNA and RNA were estimated by the method of Santen \& Agranoff (1963). The nucleotides derived from each fraction were adsorbed on charcoal and eluted, and the total phosphorus was determined. Succinoxidase was estimated manometrically by the method of Schneider \& Potter (1943). The results of representative analyses are recorded in Table 1.

Table 1. Distribution of protein, $R N A, D N A$ and succinoxidase activity in subcellular fractions from adult rat brain

The centrifugal forces used to separate the fractions were as indicated in the text. Separate homogenates were employed for the determination of protein and nucleic acids and for succinoxidase. The whole homogenate, per g. of brain tissue, contained $105.0 \mathrm{mg}$. of protein, $3.394 \mathrm{mg}$. of RNA, $3.00 \mathrm{mg}$. of DNA and utilized $3.437 \mathrm{ml}$. of $\mathrm{O}_{2} / \mathrm{hr}$. during the oxidation of succinate. The abbreviations used are as given in the text.

Distribution (\% of total in homogenate)

$\begin{array}{ccccc}\text { Fraction } & \text { Protein } & \text { RNA } & \text { DNA } & \text { Succinoxidase } \\ \mathrm{H} & (100) & (100) & (100) & (100) \\ \mathrm{N} & 11 \cdot 8 & 2 \cdot 0 & 92 \cdot 0 & \mathrm{Nil} \\ \mathrm{M}_{1} & 19 \cdot 2 & 12 \cdot 8 & \mathrm{Nil} & 42 \cdot 3 \\ \mathrm{M}_{2} & \mathbf{3 1 \cdot 9} & 21 \cdot 6 & \mathrm{Nil} & 40 \cdot 3 \\ \mathrm{M}_{3} & 10 \cdot 2 & 42 \cdot 7 & \mathrm{Nil} & \mathrm{Nil} \\ \mathrm{S}_{3} & 20 \cdot 4 & 27 \cdot 3 & \mathrm{Nil} & \mathrm{Nil}\end{array}$

Determination of enzymic activity. A solution of guanine was prepared by dissolving the solid in the minimum amount of $\mathrm{N}-\mathrm{NaOH}(1 \cdot 0-1 \cdot 2 \mathrm{ml}$. of alkali for $45 \mathrm{mg}$. of guanine) and diluting with water to give $10 \mu \mathrm{moles} / \mathrm{ml}$. The $\mathrm{pH}$ of the solution was approx. 11. Guanine-deaminase activity was determined by spectrophotometric estimation of the disappearance of substrate, by the method of Roush \& Norris (1950). The assay system was made up of the following components added in the order indicated: $0.60 \mathrm{ml}$. of $0.1 \mathrm{M}$-tris buffer, $\mathrm{pH} 8.0 ; 0.30 \mathrm{ml}$. of guanine solution ( $3 \mu \mathrm{moles}) ; 1.0 \mathrm{ml}$. of water; $0.10 \mathrm{ml}$. of enzyme preparation (whole homogenate, separated fractions, or reconstituted material). The final $\mathrm{pH}$ of the assay system was $8 \cdot 1-8 \cdot 2$. The mixture was incubated at $30^{\circ}$ for $5 \mathrm{~min}$. and deproteinized with $1.0 \mathrm{ml}$. of $10 \%(\mathrm{v} / \mathrm{v}) \mathrm{HClO}_{4}$, bringing the total volume to $3.0 \mathrm{ml}$. After centrifuging, the extinction of the suitably diluted supernatant was measured at $245 \mathrm{~m} \mu$ in a Unicam SP. 500 spectrophotometer or in a Beckman DU model G 2400 spectrophotometer, with silica cells of light-path $1 \mathrm{~cm}$. In the control experiments guanine was added after deproteinization. The decrease occurring in the extinction of the experimental sample, compared with the control, was a measure of the guanine-deaminase activity. In some experiments, the control sample was diluted 30-fold with water before extinction measurements when an extinction value of $\mathbf{0 . 4}$ was obtained. In others, the control sample was diluted 60 -fold. The experimental samples were so diluted, the minimum being 30 -fold, that the decrease in extinction was in the range $0 \cdot 02-0 \cdot 10$. Under the conditions of the experiments, the cell fractions by themselves contributed negligible absorption at $245 \mathrm{~m} \mu$. With concentration of the whole homogenate or the heavy mitochondria, as used in the standard assay system, but without added guanine, the extinction of the undiluted deproteinized samples was about $0 \cdot 2$. Hence acid-soluble tissue constituents do not interfere in the various experiments.

One unit of guanine-deaminase activity is the disappearance of $1 \mu$ mole of guanine in $5 \mathrm{~min}$./g. of fresh brain or liver or equivalent at $30^{\circ}$. In the Tables the activities are reported as mean values \pm S.D.

Comparable results were obtained when guanine-deaminase activity was assayed in some experiments in a xanthineoxidase-coupled system by spectrophotometric measurement of uric acid production (Kalckar, 1947).

\section{RESULTS}

Distribution of guanine deaminase in fractions from brain and liver. The results in Table 1 (see the Materials and Methods section) show that the procedure leads to fractionation into well-defined fractions, as judged from the distribution of DNA, RNA and succinoxidase. Slight contamination of fractions by one another may be expected with both liver and brain, but not to such a degree as to affect the validity of the general conclusions drawn below.

The results of three experiments each with rat brain and liver are presented in Tables 2 and 3.

The results show that, although the $S_{1}$ fraction contained the greater part of the activity of brain 
Table 2. Subcellular distribution of guanine-deaminase activity in rat brain

A $10 \%$ homogenate of tissue from adult male rat was prepared in $\mathbf{0 . 2 5} \mathrm{m}$-sucrose and subjected to differential centrifugation without prior treatment with Triton X-100. The volume of the supernatant fraction was adjusted to twice the volume of homogenate and that of the suspensions of the residues to half. The mixing of components was in the ratio in which they were present in the homogenate. The incubation mixture was as given in the text. The standard mean values of guanine-deaminase activity are expressed in $\mu$ moles of guanine degraded in $5 \mathrm{~min}$. $/ \mathrm{g}$. of tissue or tissue equivalent at $30^{\circ}$, and the percentage distributions on a whole homogenate basis. Reconstitution was effected in volumes proportional to the different separated fractions.

\begin{tabular}{|c|c|c|c|c|}
\hline \multirow[b]{2}{*}{ Fraction } & \multicolumn{2}{|l|}{ Control } & \multicolumn{2}{|c|}{$\begin{array}{l}\text { Triton added } \\
\text { after cell fractionation }\end{array}$} \\
\hline & $\begin{array}{c}\text { Units } \\
\text { (standard mean value) }\end{array}$ & $\begin{array}{c}\text { Distribution } \\
(\%)\end{array}$ & $\begin{array}{c}\text { Units } \\
\text { (standard mean value) }\end{array}$ & $\begin{array}{c}\text { Distribution } \\
(\%)\end{array}$ \\
\hline $\mathbf{H}$ & $30 \cdot 4 \pm 5 \cdot 1$ & $(100)$ & $63 \cdot 2 \pm 9 \cdot 8$ & $(100)$ \\
\hline $\mathbf{N}$ & $8 \cdot 5 \pm 0.2$ & 28 & $14 \cdot 1 \pm 0 \cdot 2$ & 22 \\
\hline $\mathbf{M}_{1}$ & $8 \cdot 5 \pm 0.2$ & 28 & $11 \cdot 6 \pm 0 \cdot 2$ & 18 \\
\hline $\mathbf{M}_{2}$ & $35 \cdot 0 \pm 10.5$ & 116 & $90 \cdot 9 \pm 3 \cdot 2$ & 144 \\
\hline $\mathrm{S}_{1}$ & $92.5 \pm 0.2$ & 305 & $202 \cdot 4 \pm 0 \cdot 0$ & 321 \\
\hline $\mathrm{S}_{2}$ & $84 \cdot 8 \pm 1 \cdot 9$ & 279 & $79 \cdot 4 \pm 6 \cdot 5$ & 126 \\
\hline $\mathrm{S}_{1}+\mathrm{N}$ & $85 \cdot 0 \pm 2 \cdot 4$ & 280 & $130 \cdot 3 \pm 35 \cdot 0$ & 206 \\
\hline $\mathrm{S}_{1}+\mathrm{M}_{1}$ & $64 \cdot 0 \pm 1 \cdot 5$ & 211 & $95 \cdot 0 \pm 14 \cdot 1$ & 150 \\
\hline $\mathrm{S}_{2}+\mathrm{N}$ & $85 \cdot 0 \pm 2 \cdot 4$ & 280 & $105 \cdot 0 \pm 5 \cdot 2$ & 166 \\
\hline $\mathrm{S}_{2}+\mathrm{M}_{1}$ & $42 \cdot 5 \pm 1 \cdot 2$ & 140 & $42 \cdot 0 \pm 1 \cdot 3$ & 66 \\
\hline $\mathrm{S}_{2}+\mathrm{M}_{2}$ & $100 \cdot 7 \pm 7 \cdot 4$ & 331 & $145 \cdot 9 \pm 7 \cdot 2$ & 231 \\
\hline $\mathrm{S}_{2}+\mathrm{N}+\mathrm{M}_{1}+\mathrm{M}_{2}$ & $77 \cdot 3 \pm 10 \cdot 9$ & 221 & $123 \cdot 7 \pm 10 \cdot 2$ & 196 \\
\hline
\end{tabular}

Table 3. Subcellular distribution of guanine-deaminase activity in rat liver

Details are same as given in Table $2 . R_{1}$ represents the total residue obtained at $5000 \mathrm{~g}$, composed of the nuclear and the heavy-mitochondrial fractions.

\begin{tabular}{|c|c|c|c|c|}
\hline \multirow[b]{2}{*}{ Fraction } & \multicolumn{2}{|l|}{ Control } & \multicolumn{2}{|c|}{$\begin{array}{c}\text { Triton added } \\
\text { after cell fractionation }\end{array}$} \\
\hline & $\begin{array}{l}\text { Units } \\
\text { (standard mean value) }\end{array}$ & $\begin{array}{c}\text { Distribution } \\
(\%)\end{array}$ & $\begin{array}{l}\text { Units } \\
\text { (standard mean value) }\end{array}$ & $\begin{array}{c}\text { Distribution } \\
(\%)\end{array}$ \\
\hline $\mathbf{H}$ & $14 \cdot 8 \pm 3 \cdot 1$ & $(100)$ & $28 \cdot 0 \pm 6 \cdot 1$ & $(100)$ \\
\hline $\mathbf{R}_{1}$ & $2 \cdot 1 \pm 0.0$ & 14 & $5.8 \pm 1.9$ & 21 \\
\hline $\mathrm{S}_{1}$ & $51 \cdot 0 \pm 0 \cdot 0$ & 345 & $93 \cdot 5 \pm 0 \cdot 0$ & 335 \\
\hline $\mathbf{M}_{2}$ & $12 \cdot 3 \pm 2 \cdot 5$ & 83 & $25 \cdot 6 \pm 3 \cdot 1$ & 92 \\
\hline $\mathrm{S}_{2}$ & $48 \cdot 0 \pm 4 \cdot 2$ & 325 & $51 \cdot 1 \pm 7 \cdot 0$ & 183 \\
\hline$S_{1}+R_{1}$ & $37 \cdot 0 \pm 7 \cdot 8$ & 250 & $44 \cdot 2 \pm 19 \cdot 3$ & 158 \\
\hline$S_{2}+R_{1}$ & $33 \cdot 1 \pm 10 \cdot 3$ & 224 & $35 \cdot 1 \pm 0 \cdot 2$ & 129 \\
\hline $\mathrm{S}_{2}+\mathrm{M}_{2}$ & $51 \cdot 0 \pm 0 \cdot 0$ & 345 & $93 \cdot 2 \pm 0 \cdot 0$ & 333 \\
\hline
\end{tabular}

and liver homogenates, the two particulate fractions, $\mathbf{N}$ and $\mathbf{M}_{1}$ in brain, retained some activity, together constituting about $15 \%$ of the activity of the supernatant. The $15000 \mathrm{~g}$ supernatants $\left(\mathrm{S}_{2}\right)$ of both brain and liver homogenates were only slightly less active than the $5000 \mathrm{~g}$ supernatants. Light mitochondria from brain had one-third of the activity of supernatant $S_{1}$ and those from liver about one-quarter. The two light-mitochondrial fractions had almost the same activity as the whole homogenates. Treatment with Triton X-100 increased 1.5-2-fold the activity of the whole homogenate and isolated fractions $N$ and $M_{1}$ of brain. Triton X-100 increased the activity of brain light mitochondria over $\mathbf{2 \cdot 5}$-fold and doubled the activity of the liver fraction. The increase occurring in $S_{1}$ fraction of brain on treatment with Triton $\mathrm{X}-100$ was in the same range as the activity of the detergent-treated mitochondria, but was much higher in the liver fraction. The activity of the $15000 \mathrm{~g}$ supernatant was not affected by treatment with Triton X-100. The mixed nuclear and heavymitochondrial fractions $\left(R_{1}\right)$ from liver sedimenting at $5000 \mathrm{~g}$ contained about one-seventh of the activity of the homogenate.

In the reconstitution experiments in rat brain, some inhibition occurred on mixing the nuclear fraction with the $S_{1}$ supernatant fraction. The 
guanine-deaminase activity of fraction $S_{2}$ was inhibited by the addition of any of the particulate fractions; the nuclear fraction inhibited by less than $10 \%$, and the light-mitochondrial fraction by about $15 \%$, whereas the heavy-mitochondrial fraction inhibited by $50 \%$. When fraction $\mathrm{S}_{2}$ was mixed with all the three particulate fractions, the resulting activity was about $40 \%$ less than the sum of the individual activities. However, the activity of this completely reconstituted system was about $2 \cdot 5$-fold higher than that of the whole homogenate. After treatment with detergent, the nuclear fraction did not affect the $S_{2}$ fraction. Both the light and heavy mitochondria inhibited, the former by $20 \%$ and the latter by as much as $50 \%$. The activity of the fully reconstituted system was about $30 \%$ less than the sum of the individual activities, but it was still 2 -fold higher than the activity of the whole homogenate treated with Triton X-100.

When the supernatant fraction $S_{1}$ or $S_{2}$ of rat liver was mixed with fraction $R_{1}$, the activity was $30-35 \%$ less than the calculated sum. When fraction $\mathrm{S}_{2}$ was mixed with light mitochondria, the inhibition was less. Treatment with detergent before mixing resulted in about $50 \%$ inhibition of supernatant $S_{1}$ and about $40 \%$ of fractions $S_{2}$ by fraction $R_{1}$. There was a slight increase in the additive activity on mixing fraction $S_{2}$ with the light-mitochondrial fraction. The activity of the mixture was the same as that of detergent-treated fraction $S_{1}$, agreeing with the results obtained with untreated fractions.

The results of three different experiments each on rat brain and liver, where further fractionation into microsomal fraction $M_{3}$ and supernatant $S_{3}$ was effected, are presented in Table 4.

The combined residue of nuclear, heavy mitochondria and light mitochondria $\left(\mathbf{R}_{2}\right.$ fraction) did not show any activity in brain, but had about onesixth of the activity of whole homogenate in liver. Treatment of the residue with Triton X-100 resulted in the exposure of significant activity in the brain fraction and a doubling of activity in the liver fraction. The (unwashed) microsomal residue $M_{3}$ of brain and liver contained about 40 and $65 \%$ respectively of the activity of the corresponding whole homogenates. The $S_{3}$ fraction had a lower activity than fraction $S_{2}$, the difference being almost the same as the activity of the microsomal pellet. The activity of fraction $S_{3}$ was only about $30 \%$ higher than that of the whole homogenate in brain, but was about 2.5-fold higher in liver. A combined residue of nuclear, heavy-mitochondrial, lightmitochondrial and microsomal fractions $\left(R_{3}\right.$ frac. tion) did not show any activity in both brain and liver, but treatment of these fractions with detergent exposed activity. The microsomal fraction did not seem to be affected by the detergent; the $S_{2}$ and the $S_{3}$ supernatant fractions underwent a slight inhibition.

In brain, there was some activation when fraction

\section{Table 4. Subcellular distribution of guanine-deaminase activity in rat brain and liver}

$R_{2}$ is constituted of nuclear, heavy-mitochondrial and light-mitochondrial material obtained at $15000 \mathrm{~g} ; \mathrm{R}_{3}$ is the total residue composed of nuclear, heavy-mitochondrial, light-mitochondrial and microsomal $\left(\mathrm{M}_{3}\right)$ fractions obtained at $14000 \mathrm{~g} . \mathrm{S}_{3}$ is the supernatant at $140000 \mathrm{~g}$. Other details are as given in Table 2.

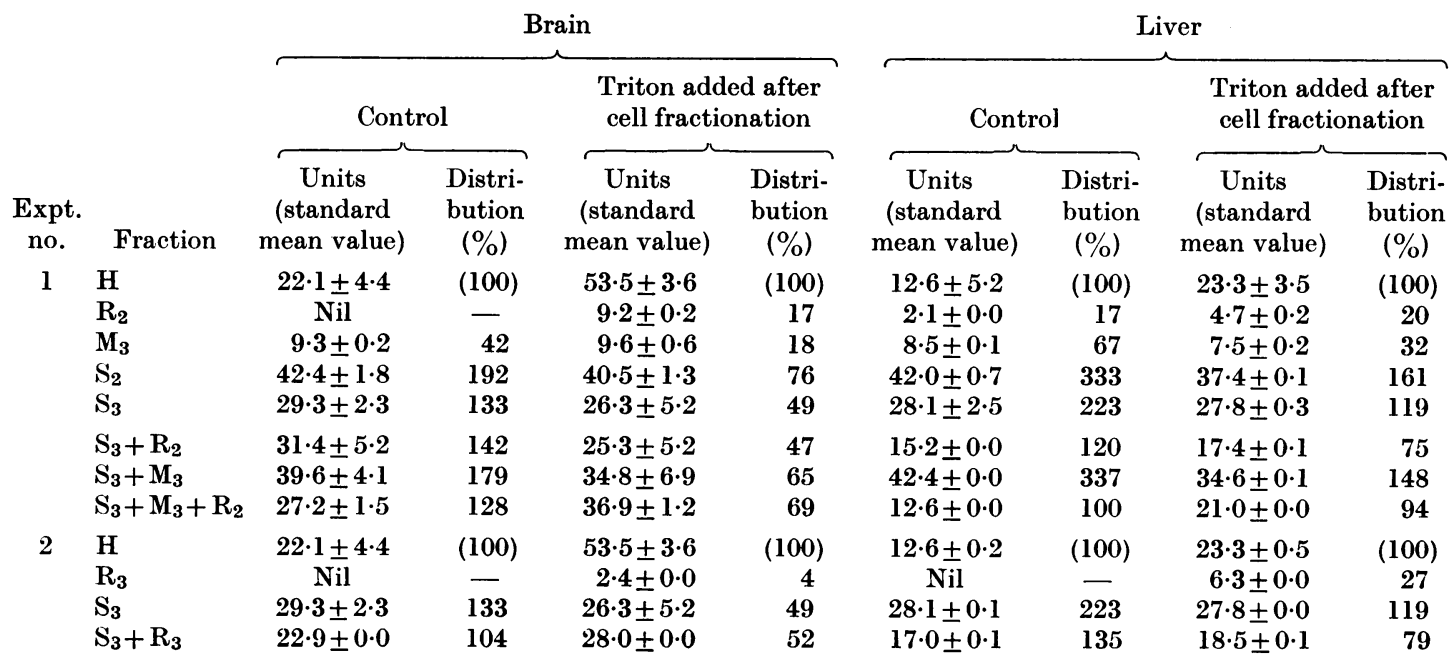


$S_{3}$ was mixed with fraction $R_{2}$, whereas on mixing with fraction $R_{3}$ there was about $22 \%$ inhibition. However, mixing of fraction $\mathbf{S}_{3}$ with fractions $\mathbf{R}_{3}$ and $R_{2}$ in liver brought about nearly 40 and $50 \%$ inhibition respectively. There was no change in the additive activity on mixing fraction $S_{3}$ with the microsomal pellet in brain, but there was about $16 \%$ increase in liver. Mixing of fraction $S_{3}$ with the microsomal and the $R_{2}$ fractions brought the activity of the fully reconstituted system to the same level as that of the whole homogenate in liver, but there was about a $23 \%$ increase in brain. When the mixing with fraction $S_{3}$ was done after prior exposure to detergent, the $R_{2}$ fraction inhibited by about $30 \%$ in brain and by $46 \%$ in liver. The microsomal pellet was without any significant effect on the $\mathbf{S}_{3}$ fraction. The $\mathbf{R}_{3}$ fraction did not have any effect on mixture with fraction $S_{3}$ in brain, whereas it resulted in about $45 \%$ inhibition in liver. However, the fully reconstituted system $\left(S_{3}+R_{2}+\right.$ microsomes or $\mathrm{S}_{3}+\mathbf{R}_{3}$ ) showed pronounced inhibition, the resulting activity being, in fact, lower than the activity of the detergent-treated whole homogenate of both brain and liver.

Statistical evaluation showed the results expressed in the preceding sections to be significant.

Nature of inhibitor. The heavy mitochondria were used as the source of inhibitor. The activity of the inhibitor was tested against the $15000 \mathrm{~g}$ supernatant, which, as shown above, was not influenced much in its guanine-deaminase activity by the addition of Triton X-100. In experiments aimed at effecting maximum exposure of inhibitory activity, the heavy-mitochondrial fraction was subjected to high-speed homogenization or treatment with ultrasonics, and tested as such or after treatment with Triton X-100. The suspension of heavy mitochondria in sucrose was homogenized in the micro-cup of a VirTis homogenizer (supplied by Arthur H. Thomas Co.) with the dial set at 50, for a total of $2 \mathrm{~min}$., with a $1 \mathrm{~min}$. interval, the suspension being kept chilled by externally packing with ice. Ultrasonic disintegration was effected at $25 \mathrm{kcyc}$./sec. for $10 \mathrm{~min}$. in the chilled stainlesssteel cup of a Mullard low-frequency apparatus with output and cathode currents of 2.5 and $0.275 \mathrm{~A}$ respectively. The percentage inhibition of guanine deaminase brought about by equivalent amounts of the treated fractions are presented in Table 5. In calculating the inhibition the apparent guaninedeaminase activity present in the mitochondrial preparations was also taken into account.

It was evident that ultrasonic disintegration or VirTis homogenization of heavy mitochondria, followed by treatment with Triton X-100, resulted in maximum exposure of the guanine-deaminase inhibitor. Ultracentrifugation at $140000 \mathrm{~g}$ of the
Table 5. Exposure and solubilization of guaninedeaminase inhibitor of heavy mitochondria

The $15000 \mathrm{~g}$ supernatant fraction used in the experiments as source of guanine deaminase contained 0.68 unit of activity.
Treatment of mitochondria

Fresh

VirTis-disrupted

Ultrasonically disintegrated

VirTis-disrupted and Triton-treated

Ultrasonically disintegrated and Triton-

treated

Supernatant of VirTis-homogenized Tritontreated
Triton-treated

\section{Percentage inhibition of total guanine- deaminase activity}

18

31

43

50

61

61

62
VirTis-homogenized Triton-treated mitochondria led to the complete recovery of the inhibitor in the supernatant, indicating true solubilization. Experiments with the supernatant fraction revealed that the inhibitor was precipitated by trichloroacetic acid and by ammonium sulphate at 0.5 saturation. It was non-diffusible and was completely inactivated by heating at $50^{\circ}$ for $5 \mathrm{~min}$.

\section{DISCUSSION}

The greater part of guanine deaminase was in the soluble cytoplasmic fraction in both brain and liver. Whereas the whole sediments from brain and liver homogenates at $140000 \mathrm{~g}$ did not show guaninedeaminase activity, the particulate fractions resulting on differential centrifugation possessed activity. The light-mitochondrial fraction was especially rich in the enzyme. Guanine deaminase therefore belongs to the class of enzymes occurring in more than one single intracellular site (de Duve, Wattiaux \& Baudhuin, 1962).

The heavy-mitochondrial fractions were characterized by the capacity to inhibit the guaninedeaminase activity of the supernatant fractions. Cell fractionation by differential centrifugation led to the concentration of inhibitor in some particles, permitting the recovery of much more enzyme activity than was demonstrable in the whole homogenate. In many respects the distribution pattern of guanine deaminase resembled that of pyridoxal phosphokinase in mammalian tissue (McCormick, Gregory \& Snell, 1961). There was some resemblance also to the distribution of ribonuclease activity (Roth, 1956), but in this case the inhibitor occurred in the soluble fraction.

Bioch. 1965, 95 
The existence of latent enzymes and their exposure by detergent action is a well-recognized phenomenon (Wattiaux \& de Duve, 1956; Bendall \& de Duve, 1960). The guanine deaminase associated with particulate material may be considered to exist partly in the exposed and the rest in the latent form. The inhibitor would also appear to exist in the exposed and latent forms in the heavy mitochondria, but almost entirely in the latent form in the nuclear fraction.

There are several aspects still awaiting elucidation. Complete reconstitution by mixing the $S_{3}$ fraction with the isolated particles did not, in general, decrease the activity of the system to the same level as the homogenate. Although mixing of the light-mitochondrial and the $S_{2}$ supernatant fractions restored the activity to that of the $S_{1}$ fraction, the sum of the activities of the two isolated components exceeded the activity of fraction $S_{1}$. The heavy-mitochondrial fraction and detergenttreated nuclear fraction had demonstrable guaninedeaminase activity, although they brought about considerable inhibition of the activity of the supernatant fractions. The presence of a distinct particle-bound enzyme unaffected by the inhibitor is a possibility. The increase of enzymic activity in whole homogenate and the appearance of activity in the whole residues at $15000 \mathrm{~g}$ and $140000 \mathrm{~g}$ on treatment with detergent are probably to be ascribed to the release of latent enzyme exceeding that of inhibitor, the detergent releasing inhibitor-insensitive enzyme from particles, or both. The actual separation of the particulate material probably facilitates a more effective exposure of inhibitory material by Triton X-100 than when the organelles are present along with the cytoplasmic fluid in the whole homogenate. This would explain why the activity of the mixture of detergent-treated $S_{3}$ supernatant fraction and total residue is lower than that of detergent-treated whole homogenate, even assuming that the enzyme component released from the nuclear and the heavy-mitochondrial material was unaffected by inhibitor. At the same time, it must be pointed out that the postulate of the existence of inhibitor- insensitive enzyme component in some of the particles renders difficult the understanding of the inactive nature of the $R_{2}$ and $R_{3}$ residues not subjected to treatment with Triton X-100.

The significance of the broad spectrum of intracellular distribution of guanine deaminase in mammalian tissue, of the partial latency of the activity in some particulate fractions and the existence of an inhibitory factor, in part latent and in part exposed, can only be surmised. Numerous biochemical mechanisms seem to require the presence of two or more distinct intracellular sites for a given enzyme. More detailed investigations are needed to decide whether guanine deaminase of animal tissues constitutes another example of a multiple-enzyme system (Kaplan, 1963). The presence of a naturally occurring inhibitor would constitute a means of physiological regulation of metabolism.

S. K. is grateful to the Council of Scientific and Industrial Research, New Delhi, for the award of a fellowship. The authors thank the Director, Central Drug Research Institute, Lucknow, for providing special laboratory facilities. This Laboratory is indebted to the Rockefeller Foundation for generous aid.

\section{REFERENCES}

Bendall, D. S. \& de Duve, C. (1960). Biochem. J. 74, 444. de Duve, C., Wattiaux, R. \& Baudhuin, P. (1962). In Advances in Enzymology, vol. 24, p. 291. Ed. by Nord, F. F. New York: Interscience Publishers Inc.

de Lamirande, G., Allard, C. \& Cantero, A. (1958). Cancer Res. 18, 952.

Kalckar, H. M. (1947). J. biol. Chem. 167, 461.

Kaplan, N. O. (1963). Bact. Rev. 27 (no. 2), 155.

McCormick, D. B., Gregory, M. E. \& Snell, E. E. (1961). J. biol. Chem. 236, 2076.

Roth, J. S. (1956). Biochim. biophys. Acta, 21, 34.

Roush, A. \& Norris, E. R. (1950). Arch. Biochem. 29, 124. Santen, R. J. \& Agranoff, B. W. (1963). Biochim. biophys. Acta, 72, 251.

Schneider, W. C. \& Potter, V. R. (1943). J. biol. Chem. 149, 217.

Talwar, G. P., Goel, B. K., Mansoor, M. \& Panda, N. C. (1961). J. Neurochem. 8, 310.

Wattiaux, R. \& de Duve, C. (1956). Biochem. J. 63, 606. 